\title{
Two interesting cases of left atrial myxoma
}

\author{
Alurkar VM'1 ${ }^{1}$ Neupane $\mathbf{S}^{2}$ \\ ${ }^{1}$ Professor, Cardiologist, Head of Department, Internal Medicine, ${ }^{2}$ Intern, Manipal Teaching Hospital, Pokhara, Nepal.
}

\begin{abstract}
Atrial myxoma is the most common type of benign primary heart tumours. Most of these tumours are sporadic in origin occurring at 3rd to 6th decade of life, while hereditary forms do exist. They are mostly found in the left atrium followed by right atrium and ventricles. A left atrial myxoma may present with features of mitral valve obstruction, embolic phenomenon or systemic disease. On diagnosis, prompt surgical removal is necessary which usually gives a complete cure, though recurrences have been reported. We hereby present two cases of solitary left atrial myxoma.
\end{abstract}

Key words: atrial myxoma, echocardiography, tumour impaction

P rimary heart tumours are rare with an incidence ranging between 0.0017 to $0.28 \%$. About $75 \%$ of these are benign and $50 \%$ of the benign tumours are myxoma. Myxomas are usually seen in the $3^{\text {rd }}$ to $6^{\text {th }}$ decade. Here we report two cases of solitary left atrial myxoma in two unusual age groups.

\section{Case report}

\section{Case 1}

A 17 year old female presented with complaint of increasing dyspnoea on exertion since last one year. On examination a rough rumbling mid-diastolic murmur was heard in the mitral area along with a soft systolic murmur. There was no postural variation in the quality of murmur. P2 was loud suggestive of pulmonary hypertension. Chest-X-ray showed mild cardiomegaly while the electrocardiogram (ECG) was normal. A transthoracic echocardiography was done which showed a solitary well defined, highly mobile, lobulated mass in the left atrium which was attached via a small pedicle to the inter atrial septum. The mass entered the left ventricle in diastole (Fig.1) and back to the left atrium during systole (Fig.2) and interfered with the closing of the mitral valve leaflet leading to grade 2 mitral regurgitation (Fig.3). Moderate pulmonary hypertension with functional tricuspid regurgitation was present but all the valves were intact. There was no sign suggestive of any syndrome and family history was negative for similar complaints. A diagnosis of left atrial myxoma was made and patient was referred for surgical removal of the myxoma.

\section{Case 2}

A 79 year old female presented with an acute episode of sudden onset breathlessness, not associated with cough or fever. On examination she was pale, had blood pressure of 120/70 $\mathrm{mm} \mathrm{Hg}$ and pulse-100 bpm with occasional ectopics. On auscultation there were bilateral basal crackles, wheeze and a mid diastolic murmur over the mitral area with loud S1 which was more in standing position than on supine. Chest X-Ray revealed features of left atrial enlargement and evidence of pulmonary oedema. ECG showed Q wave in leads II, III, aVF and multiple atrial ectopics. Echocardiography showed a large pedunculated mass in left atrium with irregular margins attached to atrial septum at fossa ovalis (Fig.4,5) and mild mitral regurgitation. The left ventricle and right ventricle were normal in size and contractility. The patient for the past one year was being treated in the lines of Chronic Obstructive Pulmonary Disease (COPD) for breathlessness. She was diagnosed as a case of left atrial myxoma, and the pulmonary oedema was attributed to tumour impacting the mitral valve orifice which resolved spontaneously. The patient improved with symptomatic management of pulmonary oedema and was referred for urgent surgical removal of the left atrial myxoma.
Correspondence

Dr. Shristi Neupane

Intern, Manipal Teaching Hospital

Pokhara, Nepal

E-mail: shristineupane@hotmail.com 


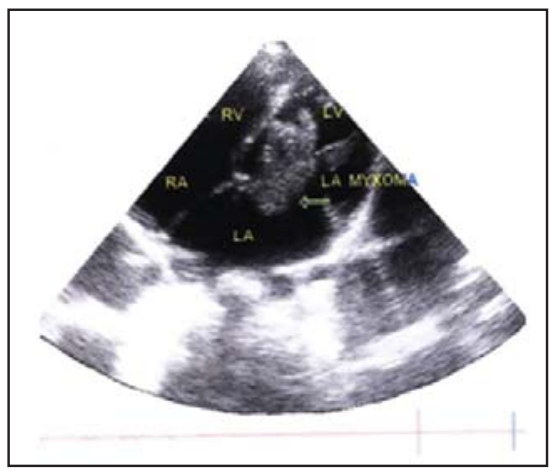

Fig 1: Left atrial myxoma in diastole

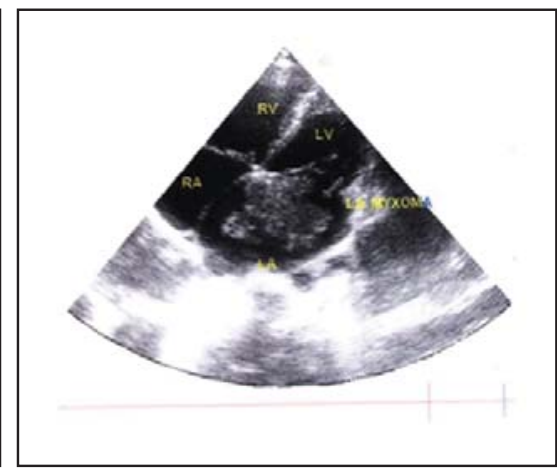

Fig 2: Left atrial myxoma in systole

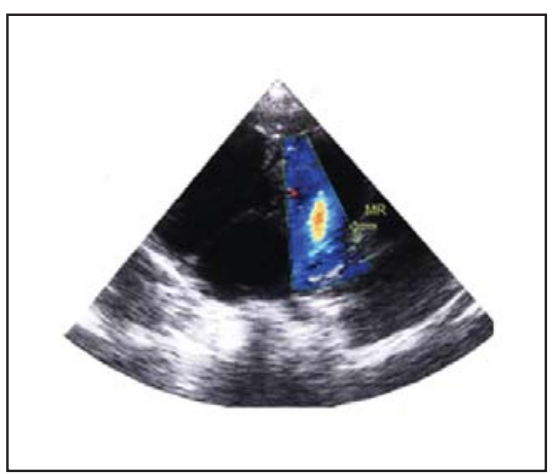

Fig 3: Mitral regurgitation.

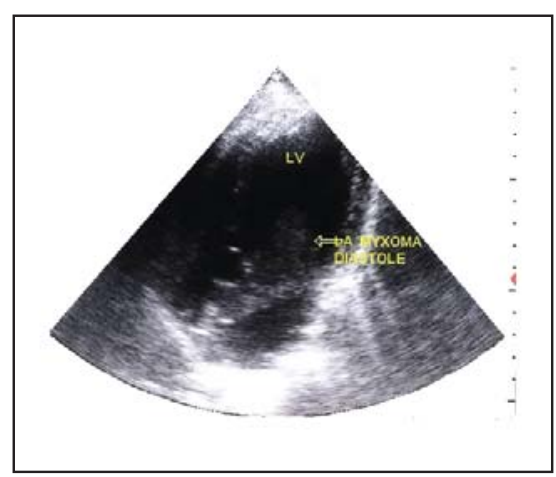

Fig 4: Left atrial myxoma in diastole

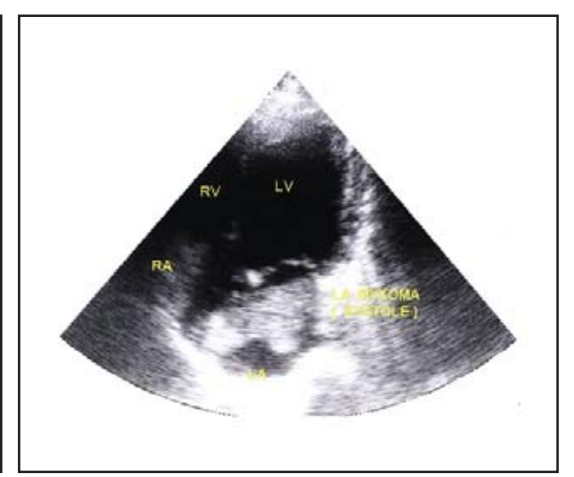

Fig 5: Left atrial myxoma in systole

\section{Discussion}

Left atrial myxomas, first described in $1845^{1}$, are sporadic tumours occurring between $3^{\text {rd }}$ to $6^{\text {th }}$ decades of life. However, $10 \%$ cases are hereditary with autosomal dominant inheritance where the patient may present with multiple myxomas at a young age. Those are usually found to be associated with various syndromes: Carney complex (myxoma of skin/breast/ heart, lentigines/pigmented nevi and endocrine over activity); LAMB syndrome (lentigines, atrial myxoma, blue nevi) and NAME syndrome (nevi, atrial myxoma, myxoid neurofibroma, and ephelides).

A left atrial myxoma may be asymptomatic if small or may present with features of mitral valve obstruction, embolic phenomenon or other systemic features like fever, weight loss, cachexia, malaise, arthralgia, rash, clubbing, anaemia, Reynaud's phenomenon etc. They usually have a short history with dramatic progression but cases have been reported where patient had slowly progressive symptoms for about 12 years ${ }^{2}$. Sometimes their only presentation may be an embolic phenomenon like an ischemic stroke in a young person. Hence patients have been frequently misdiagnosed as infective endocarditis, collagen vascular disorder, paraneoplastic syndromes or even COPD as in the case above. In patients with suspected mitral valve disease the incidence of myxoma is $0.27 \%^{3}$. Few characteristics which may help in differentiating myxoma from mitral stenosis are presence of a characteristic low pitched tumour plop during early or mid diastole, murmur with postural variation and postural syncope in a patient with features of mitral valve obstruction.

Diagnosis is usually made by echocardiography where we can see a mobile, lobulated, homogenous mass attached via a pedicle to the left interatrial septum, the commonest area being the lower end of fossa ovalis, but it may be attached to the posterior or anterior atrial wall or even the atrial appendage. It is important to look for other such masses in the heart and the state of the heart valves which may suggest damage due to myxoma or some other pre-existing pathology. A CT-Scan and MRI may help in getting further details before surgery. Earlier, cardiac catheterization and angiography were routinely done which showed the feeding vessels and signs of neovascularization ${ }^{4}$. Nowadays it is only done in patients presenting with symptoms of coronary artery disease due to the risk of tumor embolization in the procedure. Screening echocardiography is recommended for family members in cases of young patient with multiple myxomas or if associated with syndromes. 
Once diagnosed, prompt surgical removal of the myxoma is indicated. If left alone, it can be fatal anytime due to coronary or systemic embolization or sudden impaction in the mitral valve orifice. $8 \%$ mortality has been reported in patients awaiting operation following definite diagnosis ${ }^{5}$. A case was reported in 1966 where myxoma was diagnosed incidentally during digital commisurotomy for mitral stenosis and the patient was posted for surgery later. However the patient died suddenly 36 hours after the procedure and autopsy revealed impacted myxoma at the mitral orifice ${ }^{6}$. This incidence further emphasizes the importance of prompt treatment.

Surgical removal usually gives a total cure. Recurrences are rare and usually occur within the next 48 months?

\section{Conclusion}

Left atrial myxoma is a rare condition. With its varied presentation, it is not uncommon for a patient to be misdiagnosed. It is a benign tumour but can be fatal anytime due to tumour impaction on mitral valve orifice or due to embolic episodes. Hence early diagnosis and prompt treatment is very important.

\section{References}

1. King TW. On simple vascular growths in the left auricle of the heart. Lancet. 1845;2:428-9.

2. Comer TP, Arbegast R, Schmalhorst WR. Left atrial myxoma-Diagnostic and surgical aspects. Calif Med. 1973;118:18-20.

3. Effert S, Domanig E. The diagnosis of intraatrial tumor and thrombi by the ultrasonic echo method. Ger Med Mon. 1959;4:1.

4. Van Cleemput J, Daenen W, De Geest H. Coronary angiography in cardiac myxomas: findings in 19 consecutive cases and review of the literature. Cathet Cardiovasc Diagn. 1993;29(3):217-20.

5. Hibner RW, Corbus HF, Fulmer AF. Myxoma of the left atrium presenting as an embolus to the femoral artery. Arch Surg. 1963;87:525.

6. Firor WB, Aldridge HE, Bigelow WG. A followup study of three patients after removal of left atrial myxoma five to ten years previously. J Thorac Cardiovasc Surg. 1966;51:515.

7. McAllister H, Hall R, Cooley D. Tumors of the heart and pericardium. Curr Probl Cardiol. 1999;24:57-116. 